MATEC Web of Conferences 22,01047 (2015)

DOI: $10.1051 /$ matec conf/ 20152201047

(C) Owned by the authors, published by EDP Sciences, 2015

\title{
Construction of Deterministic Measurements Matrix Using Decimated Legendre Sequences
}

\author{
Xiang Cui* \\ College of Information Science and Technology, Beijing University of Chemical Technology, Beijing, China
}

\begin{abstract}
This paper proposed and constructed a new class of deterministic measurements matrix by using decimated binary Legendre sequences for convolutional Compressed Sensing. The author proves that when the measurement matrix is constructed by a random subsampling, it can offer a stable sparse reconstruction. Besides, the simulation results shows that when a deterministic subsampler is used, the proposed matrix can also guarantee the stable reconstruction as good as random Gaussian or Bernoulli matrixes do, which are commonly used in CS.
\end{abstract}

Keywords: convolutional compressed sensing; Legendre sequences; RIP; coherence

\section{INTRODUCTION}

With the rapid development of information technology, data and information become more and more important. At the same time, as the quantity of data and information is very large, the access method of information has attracted great attention. As we know, when the amount of data is huge, if we use the traditional method to acquire the information, the Nyquist specific principle must be followed. In this method, the consumption of software and hardware resources will be great, which sometimes may overtake the biggest processing capacity that we can supply. In recent years, a new signal processing theory named Compressed Sensing (CS) [1] has been put forward, which effectively solves the predicament of previously described.

As is known to all, our modern technology-driven civilization acquires and exploits ever-increasing amounts of data [1], but most of the data that we acquire is useless. Actually, those data can be "through away" with almost no perceptual loss for us. After noticing this phenomenon, we can find a way to acquire the information that is important for us by directly measure the part of the data that will not end up being thrown away. Compressed Sensing (CS) is just a method like this, which was proposed by David L. Donoho in 2006.

As mentioned above, Compressed Sensing (CS) is a method to acquire information by measuring part of the original data. Well, most of the original data are useless data, so we call this kind of data sparse. For example: let $\mathrm{s} \in R^{N}$ be a sparse signal with at most $\mathrm{k}$ nonzero components. Let $\Phi$ be a $M \times N$ measurement matrix with $M<N$. Signals like $\mathrm{S}$ are called $k$-sparse, which can be recovered using its measurements $v=\Phi \mathrm{s} \in R^{M}$ if $\mathrm{f}=\Psi \mathrm{s}$ can be approximated by $k$ nonzero entries according to the Compressed Sensing. In order to recover the signal without missing im-

*Corresponding author: cxhanzel@,126.com portant information, two distinct questions should be considered. They are shown as follows:

1) How many measurements are necessary to reconstruct the original signal?

2) Given these measurements, what algorithms can perform the reconstruction task?

To the first question, according to the experiences of previous researches, we know that no fewer than $\mathrm{k}$ measurements will be enough to assure a stable recovery. In other words, sparse signals can be reconstructed with far less information [2]. As we know, the recovery is dual to sparse approximation. And in CS theory, if the restricted isometry property (RIP) is satisfied, robust and stable reconstruction of sparse signal will be guaranteed [1]. In this paper, our research mainly focuses on the relevant aspects of the first question.

To the second question, many researchers have proposed many kinds of reconstruction algorithms after several years of development of CS theory. Those researches pointed out that the process of sparse signal reconstruction can be converted to a problem of convex optimization. In this way, the Basis Pursuit (BP) [3] algorithm proposed by Chen, Donoho and Saunders can be used to recover the original signal. However, when using BP algorithm, people find out that it sometimes brings us high computational complexity and bad reconstruction results. In 2005, a new kind of algorithm called Matching pursuit (MP) [4] was used in CS. Tropp and Gilbert point out that matching pursuit (MP) algorithm and Orthogonal matching pursuit (OMP) [2] algorithm can guarantee a good reconstruction result with lower computational complexity. With the development and progress of CS, many algorithms based on the principle of Matching pursuit are proposed, for example: Stagewise orthogonal matching pursuit (StOMP) [5], Regularized orthogonal matching pursuit (ROMP) [6], etc. At the same time, in order to achieve a better reconstruction 


\section{MATEC Web of Conferences}

result, another kind of algorithm is proposed, which uses sparse matrix as measurements matrix taking sample. This kind algorithms include: "Chain pursuit (CP)", "Fourier sampling", "Heavg Hitters on Steroids (HHS)" and so on.

As we know, researches of compressed sensing theory focus on the following three aspects: sparse representation, construction of the measurement matrix and reconstruction algorithm. As mentioned earlier, we propose to construct a new kind of deterministic measurement matrix in this paper. For a sparse signal $\mathrm{s} \in R^{N}, \Phi$ is a $M \times N$ measurement matrix, and $\mathrm{f}=\Psi \mathrm{s}$. Let $\Theta=\Phi \Psi^{H}$ be a $M \times N$ matrix. To guarantee a stable reconstruction, $\Theta$ has to satisfy the RIP. According to paper [7], we know that if $\left(1-\delta_{k}\right)\|\mathrm{f}\|^{2} \leq\|\Theta \mathrm{f}\|^{2} \leq\left(1+\delta_{k}\right)\|\mathrm{f}\|^{2}$ for all $\mathrm{f} \in R^{N}$, where $\mathrm{f}$ is an arbitrary k-sparse vector within $R^{N}$. It is well known that $\Theta=\Phi \Psi^{H}$ obeys the RIP condition as long as $M \geq O(k \log (N / k))$ if $\Phi$ is a fully random matrix, such as the Gaussian or Bernoulli matrix [7] After years of researching, we know that a structured sensing matrix can play a better role in reconstruction. There're lots of methods to construct a structured sensing matrix, one of them is to construct $\Phi$ from a partial circulant matrix [8].

$\Phi=\frac{1}{\sqrt{M}} \mathrm{R}_{\Omega} \mathbf{A}$

Where $\mathrm{R}_{\Omega}$ is a sampling operator, and $\mathbf{A}$ is an $N \times N$ circulant matrix, which can be expressed as follows:

$$
\mathbf{A}=\left[\begin{array}{cccc}
a_{0} & a_{N-1} & \mathrm{~L} & a_{1} \\
a_{1} & a_{0} & \mathrm{~L} & a_{2} \\
\mathrm{M} & \mathrm{M} & \mathrm{O} & \mathrm{M} \\
a_{N-1} & a_{N-2} & \mathrm{~L} & a_{0}
\end{array}\right]
$$

According to convolutional Compressed Sensing, matrix A can be factorized into:

$$
\mathbf{A}=\frac{1}{\sqrt{N}} \mathrm{~F}^{H} \operatorname{diag}(\sigma) \mathrm{F}
$$

Where, $\mathrm{F}$ is an $N \times N$ FFT matrix. Let $\mathbf{a}=\left[a_{0}, a_{1}\right.$, $\left.\ldots, a_{N-1}\right]$ be the first column of A. In equation (3), $\sigma$ $=\left[\sigma_{0}, \sigma_{1}, \ldots, \sigma_{N-1}\right]^{T}$ is a vector that obtained by taking the normalized FFT of $\mathbf{a}$. And it is easy to find out that

$$
\sigma=\frac{1}{\sqrt{N}} \mathrm{Fa}
$$

And, if $\sigma$ is unimodular, $\mathbf{A}$ will definitely be a unitary matrix satisfying $\mathbf{A}^{*} \mathbf{A}=N \mathrm{I}_{N}$.

In existing works, the vector $\sigma$ can be either a random unimodular sequence or a deterministic unimodular sequence. When $\sigma$ is random, $\mathrm{R}_{\Omega}$ is also random, it is proved that if $M \geq O\left(k \log ^{5} N\right)$, the RIP of $\Phi$ will be satisfied. When $\sigma$ is deterministic, $\mathrm{R}_{\Omega}$ is a deterministic sampling operator; if $M \geq O\left(k \log ^{4} N\right)$ [8], the RIP of $\Phi$ will be held for any $\Psi$ that is orthonormal. In this paper, we construct a new kind of $\sigma$ by using a decimated binary Legendre sequence [9]. We find out that the coefficients of corresponding $\Phi$ are real-valued. It is interesting to note that the measurements matrix $\Phi$ offers similar performance as that of a Gaussian or Bernoulli operator for a wide range of signal length whenever the sampler $R_{\Omega}$ is random or deterministic. This is very significant, which implies that the decimated Legendre sequence has great potential in convolutional CS.

\section{REVIEW}

In this section, some related theories in CS and convolutional CS have been shown.

In convolutional CS, there are two ways to construct a deterministic matrix A. One way is to construct $\sigma$ firstly in the frequency-domain approach. Then $\mathbf{A}$ can be obtained from (3). It is easy to find that $\mathbf{A}$ is a unitary matrix if $\sigma$ is a unimodular sequence. The other one is to construct a first, which is the first column of $\mathbf{A}$ in the time-domain approach. Then we can obtain A from (4) and (3). However, getting a unitary $\mathbf{A}$ using the time-domain approach is not easy. Thus, we will mainly focus on the former way, in which $\sigma$ is a unimodular sequence. Besides, many applications require $\mathbf{A}$ to be a real-valued matrix, such as coded aperture imaging and Fourier optics. What's more, using a real-valued matrix will be easier for computing and data saving. To generate real sensing matrix, $\sigma$ needs to satisfy some the condition that is represented in Lemma 1.

Lemma 1: For the bipolar sequence $\sigma$, whose length is $N$, if it satisfies the equation as follows:

$\sigma_{k}=\sigma_{N-k}^{*}, 1 \leq k \leq N-1$

Where the superscript $*$ represents the complex conjugate operation, $\mathbf{A}$ in (3) will be a real-valued matrix.

Theorem 1: [8] For the matrix $\Phi$ in (1), where $\mathrm{R}_{\mathrm{\rho}}$ is a random sampling operator and $\sigma$ is unimodular. If

$\mu(\mathbf{A})=\max _{0 \leq t \leq N-1}\left|a_{t}\right|=O(1)$

Then all $k$-sparse signals in the canonical basis ( $\Psi=\mathrm{I})$ or the FFT basis $(\Psi=\mathrm{F} / \sqrt{N})$ can be stably recovered as long as $M \geq O\left(k \log ^{4} N\right)$.

We know that the RIP is an essential requirement for stable reconstruction. However, for a CS operator, it is not easy to check whether the RIP is satisfied. As an alternative, deterministic design of CS operators with small spectral norm and small column coherence are widely used in signal recovery. For simplicity, let us suppose that $\mathrm{s}$ is sparse in the canonical basis with $\Psi=\mathrm{I}$. And let us normalize $\Phi=\left[\phi_{0}, \phi_{1}, \ldots, \phi_{N-1}\right]^{T}$ so that each column of $\Phi$ has unit $l_{2}$-norm. Therefore, the reconstruction can be achieved if $k<(1+1 / v(\Phi)) / 2[2]$, where $v(\Phi)=\max _{i \neq j}\left|\phi_{i}^{H} \phi_{j}\right|$ is the column coherence of $\Phi$. According to [10], we know that if $\mathrm{s}$ follows the generic $k$-sparse model, the signs of the nonzero entries will be independent and equally likely to be +1 or -1 . Besides, their positions will be 
uniformly distributed at random. In this way, the sparse vector can be reconstructed stably using $l_{1}$-min if $v(\Phi)<O(1 / \log N)$ and $k \leq O\left(N /\|\Phi\|^{2} \log N\right)$ [10]. What's more, a similar bound as that of a random Gaussian or Bernoulli operator can be achieved. When $\Phi$ is a tight frame with $\|\Phi\|^{2}=N / M$, which means $M \geq O(k \log N)$.

\section{CONVOLUTIONAL CS USING DECIMATED LEGENDRE SEQUENCE}

This section studies the construction of $\sigma$ by using a decimated binary Legendre sequence and the sparse recovery performance of the corresponding CS operator $\Phi$. In our previous researches, we have constructed a deterministic measurements matrix using $\sigma$ formed by an original Legendre sequence, which offers a good reconstruction performance. However, the length of an original Legendre sequence must be an odd prime, which means the size of the measurements matrix is limited. This restriction will definitely limit its scope of application. In order to solve the problem, we propose to use a transform of Legendre sequence to construct $\sigma$ whose length can be any positive integer.

\subsection{Construction of Decimated Legendre Sequences}

Definition 1[9]: Let $p$ be an odd prime, and Legendre symbol is a multiplicative function with values $1,-1$ and 0 . It is a quadratic character modulo prime number $p$, which can be represented as follows:

$\left(\frac{\mathrm{a}}{p}\right)=\left\{\begin{array}{l}1, \text { a is quadratic resdue } \bmod p, a \equiv 0(\bmod p) \\ -1, a \text { is quadratic non }- \text { residue } \bmod p \\ 0, a \equiv 0(\bmod p)\end{array}\right.$

Let $\mathrm{L}_{n}=\left[\mathrm{L}_{n-1}+(2 \mathrm{n}-1)\right] \bmod p$ where $\mathrm{L}_{n}$ represents the sequence indices: $\mathrm{L}_{1}=1 \& \mathrm{n}->2$ to $(p-1)$ and $p$ is the prime length. Then Legendre sequence can be defined as follows:

$\mathrm{LS}(k)=\left\{\begin{array}{r}1, k \in\left\{\mathrm{L}_{n}\right\} \\ -1, k \notin\left\{\mathrm{L}_{n}\right\}\end{array}\right.$

Where $k \in[0, p-1]$ is a positive integer.

Let $p$ be an odd prime in the finite field $\mathrm{F}_{p}$. A binary Legendre sequence $\mathbf{x}=\left(\mathrm{x}_{0}, \mathrm{x}_{1}, \ldots, \mathrm{x}_{L-1}\right)$ of period $L$ $=p$ can be defined as follows:

$\mathrm{x}_{k}=\left\{\begin{array}{r}1, k \in\left\{\mathrm{L}_{n}\right\} \\ -1, k \notin\left\{\mathrm{L}_{n}\right\}\end{array}\right.$

Construction: We've achieved a binary Legendre sequence $\mathbf{x}$ of period $L=p$ in Definition 1. Now we construct a binary sequence $\mathrm{u}=\left[u_{0}, u_{1}, \ldots, u_{q-1}\right]^{T}$ of Length $q=\lceil L / c\rceil$, where $c$ is a positive integer that divides $L$, and $u_{k}=\mathrm{x}_{k+c-1}, 0 \leq k \leq q-1$. Then it's time to construct the vector $\sigma$ that we need. Now what we need to do is to abandon the first and the last element of $\mathbf{u}$ and rearrange the sequence in reverse chronological order as a new sequence as $\mathbf{u}$ '. We define $\sigma=\left[\mathbf{u}, \mathbf{u}^{\prime}\right]^{T}$ of Length $N=2 q-2$ as the bipolar version of $\mathbf{u}$. It's clear that the matrix $\mathbf{A}$ constructed by $\sigma$ will be a real-valued according to Lemma 1 . Based on (1) and (2), we can easily get the deterministic measurement matrix $\Phi$. Besides, if we need the length of $\sigma$ to be an odd number such as $2 q-1$, we just abandon the first element of $\mathbf{u}$ and rerrange the sequence as u' . Then $\sigma=\left[\sigma_{0}, \sigma_{1}, \ldots, \sigma_{N-1}\right]^{T}=\left[\mathbf{u}, \mathbf{u}^{\prime}\right]^{T}$ can be defined as a sequence of length $N=2 q-1$. In this way, the length of $\sigma$ can be either odd or even as long as we adjust the value of $q=\lceil L / c\rceil$, which means the restriction that $\mathrm{N}$ has to be a prime number which is lifted.

\subsection{Performance in Sparse Reconstruction}

In this subsection, we propose to discuss the sparse reconstruction of $\Phi$ that we construct when $\Psi=\mathrm{I}$ or $\Psi=1 / \sqrt{N} \mathrm{~F}$

When $R_{\Omega}$ is a random sampling operator, we firstly need to check whether the RIP is satisfied. Consider $\sigma$ and $\mathbf{A}$ given in subsection 3.1. Then:

$\mu(\mathrm{A})< \begin{cases}\sqrt{c+\frac{1}{N}}+\frac{1}{\sqrt{N}}, & \text { if } \mathrm{N} \text { is even, } \\ \sqrt{c+\frac{1}{N}}, & \text { if } \mathrm{N} \text { is odd. }\end{cases}$

It's clear that $\mu(\mathrm{A})<\sqrt{ } c$ if $N$ is sufficiently large. So for the $M \times N$ matrix $\Phi$ that we construct, when $\mathrm{R}_{\Omega}$ can satisfy the RIP, if $M \geq O\left(k \log ^{4} N\right)$, the stable reconstruction can be guaranteed when $\Psi=\mathrm{I}$ or $\Psi=1 / \sqrt{N} \mathrm{~F}$.

When $\mathrm{R}_{\Omega}$ is a deterministic sampling operator, in which $\Omega=\{0,1, \ldots, M-1\}$. In this part, we propose to use the results of simulation about column coherence of different CS operators to discuss the reconstruction performance of $\Phi$ after deterministic sampling. For easy to computing, let $\Psi=\mathrm{I}$, and signal length $N$ :

$N \in\{121,156,256,365,644,871,1200,1641$,

2456, 3661, 47704, 6097, 8404$\}$.

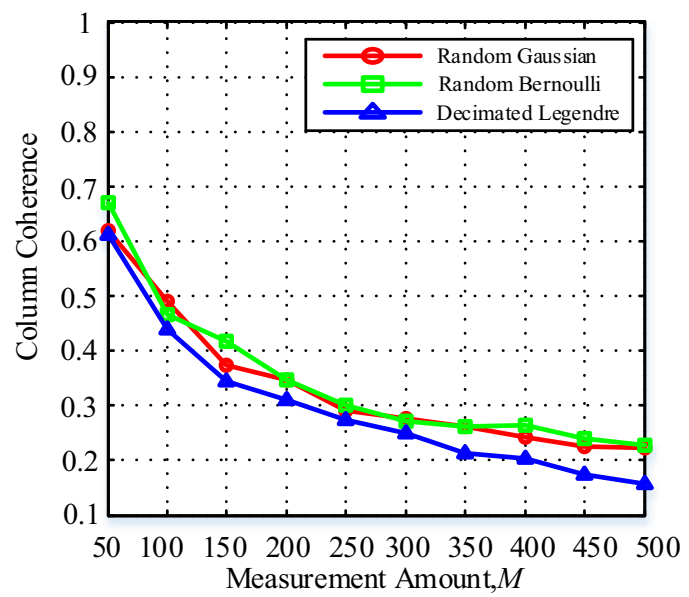

Figure 1. Column coherence of different CS operators where $\mathrm{N}=1200$. 


\section{MATEC Web of Conferences}

In this experiment, the values of $N$ include even numbers and odd numbers in order to prove that the length of $\sigma$ can be a number which is not a prime number. After simulation, we check $\|\Phi\|^{2}$ and $v(\Phi)$ for each of the values that $N$ can obtain. The simulation results of signal length $N=1200$ is showed in Fig.1.

In Figure 1, the column coherence of $\Phi$ with $N=$ 1200 constructed from a decimated Legendre sequence ( $p=1201$ and $c=2)$ shows the similar performance with that of the random Gaussian or Bernoulli matrixes. According to our simulations, $\Phi$ constructed in subsection 3.1 is nearly an incoherent tight frame with $\|\Phi\|^{2} \approx N / M, v(\Phi)<O(\sqrt{\log N} / \sqrt{M})$.

It means that the stable reconstruction can be guaranteed if $M \geq O(k \log N)$. In next section, extensive simulations will be carried out, which will give a further clarification for the performance of the measurements matrix constructed by us.

\section{SIMULATION RESULTS}

In this section, we present the simulation results for the following two experiments:

Experiment 1: In this experiment, we take a $k$-sparse signal $\mathbf{x}$ of length $N$ as the original signal, where $k=$ $N / 10$. By using OMP algorithm, we precisely check SNR $(\mathrm{dB})$

$$
S N R=10 \times \lg \frac{\|\hat{\mathrm{x}}\|_{2}^{2}}{\|\mathrm{x}-\hat{\mathrm{x}}\|_{2}^{2}}
$$

and compatibility $\alpha$

$$
\alpha=1-\frac{\|\hat{\mathrm{x}}\|_{2}-\|\mathrm{x}\|_{2} \mid}{\|\hat{\mathrm{x}}\|_{2}+\|\mathrm{x}\|_{2} \mid}
$$

Table 1. Output SNRs and compatibility in sparse signal

\begin{tabular}{|c|c|c|c|}
\hline & $\mathrm{R}_{\Omega}$ & $\mathrm{SNR} / \mathrm{dB}$ & $\begin{array}{l}\text { Compatibility } \\
\alpha\end{array}$ \\
\hline \multirow[t]{2}{*}{$\begin{array}{l}\text { Proposed } \\
\Phi\end{array}$} & $\begin{array}{l}\text { Random } \\
\text { Sampling }\end{array}$ & 33.77 & 0.9971 \\
\hline & Deterministic & 30.21 & 0.9863 \\
\hline \multicolumn{2}{|c|}{ Random Gaussian Marix } & 33.96 & 0.9975 \\
\hline \multicolumn{2}{|c|}{$\begin{array}{l}\text { Random Bernoulli Ma- } \\
\text { trix }\end{array}$} & 34.29 & 0.9985 \\
\hline
\end{tabular}
reconstruction.

Table 1 shows the reconstruction performance of $\Phi$ with $N=1200, M=0.3 N$ constructed from a decimated Legendre sequence $(p=1201$ and $c=2)$, which also include the reconstruction performance of a random Gaussian operator or a random Bernoulli operator. The reconstruction results are shown in Figure 2. The simulations indicate that $\Phi$ constructed in Section 3 can supply the similar reconstruction performance to that of a random Gaussian or Bernoulli matrix.
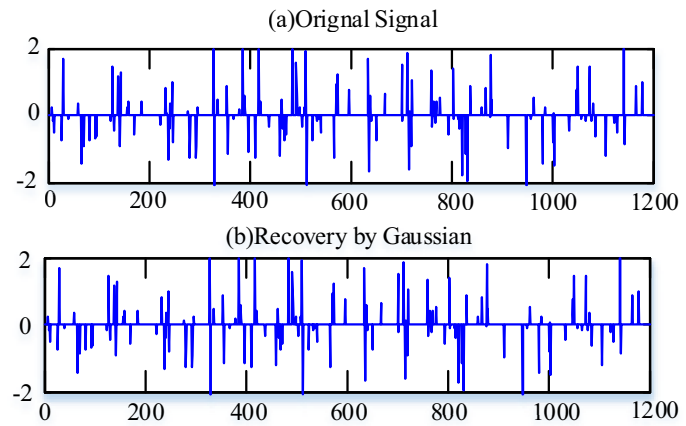

(c)Recovery by Bernoulli

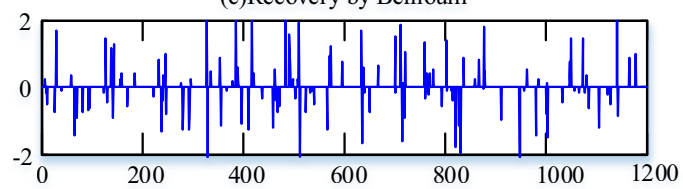

(d)Recovery by Proposed matrix after random subsampling
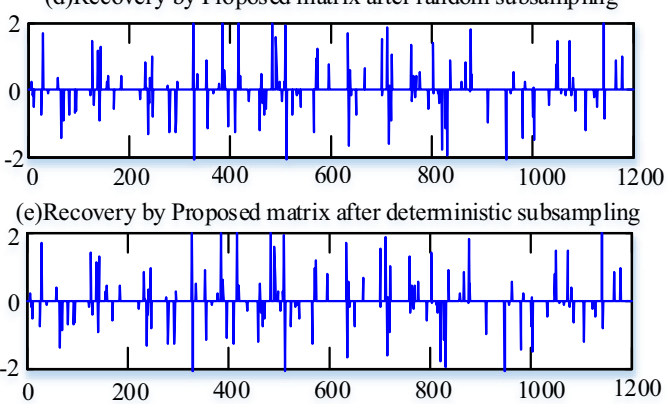

Figure 2. Reconstruction results for $\mathrm{N}=1200, \mathrm{M}=0.3 \mathrm{~N}=$ 360

Experiment 2: In this experiment, we study the application of the proposed measurements matrix in reconstruction of imaging of $2 \mathrm{D}$ signals. And in this application, A needs to be real-valued. The reconstruction algorithm of OMP is applied here. As shown in Figure 3, three 8-bit, 256 $\times 256$ images have been used. Figure 3 (a) "Lena" is a portrait image, Figure 3(f) "Stars" is an astronomical image, and Figure 3(k) "Brain" is a medical image. The measurement matrixes used in this experiment are all $M \times N$ matrix $(N=$ $256, M=0.6 N)$. Besides, the reconstructed SNRs are also showed in Fig. 2. In Fig. 2, the "D" in "D-Gaussian" represents "deterministic", which means the measurement matrix is constructed by deterministic subsampling. And, the "R" in "R-Gaussian" represents "Random".

The simulation results indicate that the proposed deterministic measurement matrix can offer similar (even better performance in some conditions) performance as that of some commonly-used random measurement matrix, such as Gaussian and Bernoulli matrix, both in terms of the SNRs and the visual quality of reconstructed images. 


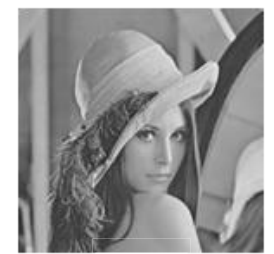

(a)

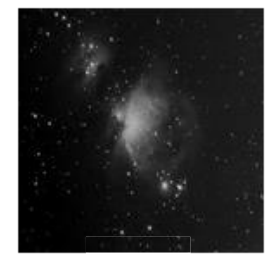

(f)

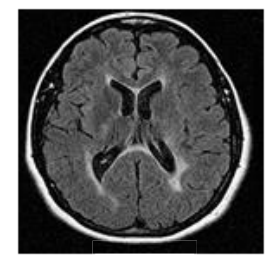

(k)

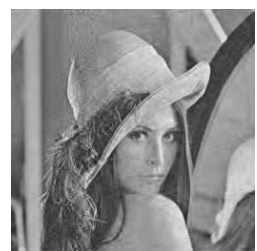

(b)

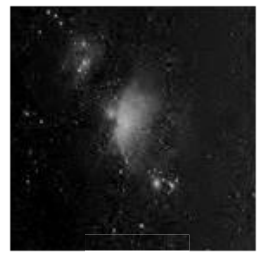

(g)

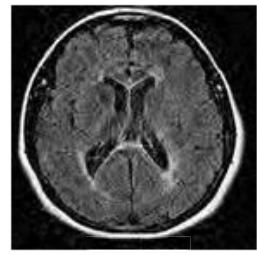

(1)

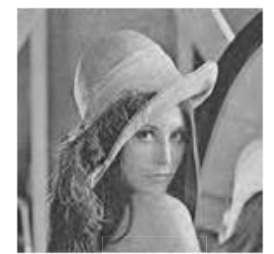

(c)

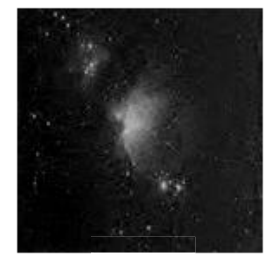

(h)

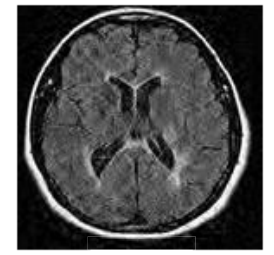

(m)

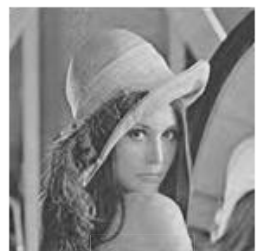

(d)

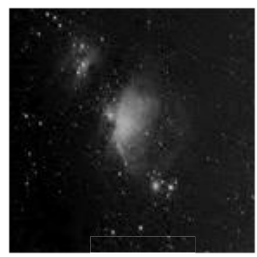

(i)

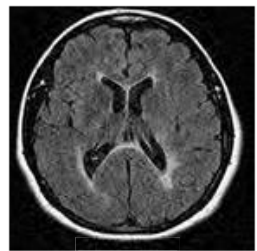

(n)

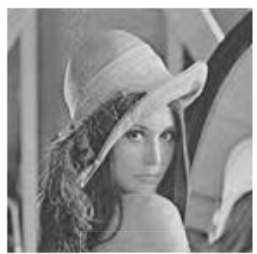

(e)

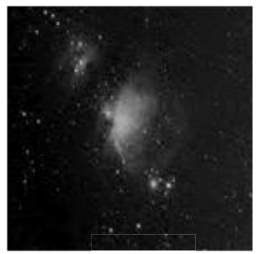

(j)

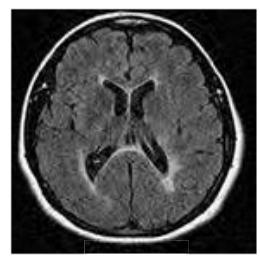

(o)

Figure 3. Original images and reconstructed results

First row, "Lena" images. (a) Original image. (b) R-Gaussian: $30.89 \mathrm{~dB}$; (c) D-Gaussian: $30.81 \mathrm{~dB}$ (d) R-DL: $32.80 \mathrm{~dB}$; (e) D-DL: $32.80 \mathrm{~dB}$. Second row, "Star" images. (f) Original image. (g) R-Gaussian: $30.38 \mathrm{~dB}$; (h) D-Gaussian: $30.25 \mathrm{~dB}$; (i) R-DL: $31.83 \mathrm{~dB}$; (j) D-DL: 31.74dB. Third row, "Brain" images. (k) Original image. (1 R-Gaussian: $23.56 \mathrm{~dB}$; (m) D-Gaussian: $23.37 \mathrm{~dB}$; (n) R-DL: $24.66 \mathrm{~dB}$; (o) D-DL: 24.53d

\section{CONCLUSION}

In this paper, a new class of deterministic measurement matrixes have been proposed, which were constructed from a decimated Legendre sequence. It has been validated that no matter the matrix is constructed by a random subsampling or a deterministic susampling, it can guarantee a stable sparse signal reconstruction. What's more, its reconstruction performance is as good as (sometimes even better than) that of fully random Gaussian matrix. Our analysis shows that the new CS measurement matrixes from decimated Legendre sequences that can offer reliable reconstruction performance are simple to construct. In convolutional CS, they may have more potential applications.

\section{REFERENCES}

[1] Donoho, D.L. 2006. Compressed sensing. IEEE Trans. Inf. Theory, 52(4): 1289-1306.

[2] Tropp, J.A. \& Gilbert, A.C. 2007. Signal recovery from random measurements via orthogonal matching pursuit. IEEE Trans. Inf. Theory, 53(12): 4655-4666.
[3] Chen, S.B. Donoho, D.L. \& Saunders, M.A. 1998. Atomic decomposition by basis pursuit. SLAM Review, 20(1): 33-61

[4] Mallat, S. \& Zhang, Z. 1993. Matching pursuit with time-frequency dietionaries. IEEE Trans Signal Process, 41(12): 3397-3415.

[5] Donoho, D.L. Tsaig, Y. Drori, I. \& etc. 2006. Sparse solution of underdetermined linear equations by stagewise orthogonal matching pursuit. Technical Report.

[6] Needell, D. \& Vershynin, R. Signal recovery from incomplete and inaccurate measurements via regularized orthogonal matching pursuit [online].

[7] Candes, E.J. \& Tao, T. 2006 Near-optimal signal recovery from random projections: Universal encoding strategies. IEEE Trans. Inf. Theory, 52(12): 5406-5425.

[8] Li, K.Z. Gan, L. \& Ling, C. 2013. Convolutional compressed sensing using deterministic sequences. IEEE Trans. Inf. Theory, 61(3): 740-752.

[9] Ravikumar, C. \& Sudha, K. 2014. Legendre and polyphase sidel'nikov sequence for applications in space communication. LJESE, 2(9): 2319-6378.

[10] Yu, N.Y. \& Gan, L. 2014. Convolutional compressed sensing using decimated sidelnikov sequences. IEEE Signal Processing Letters, 21(5): 591-594. 